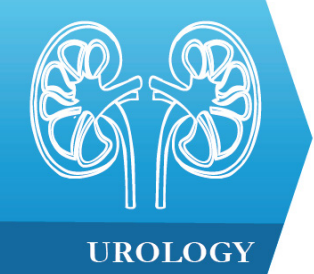

1) $2^{\text {nd }}$ Department of ObstetricsGynecology, Iuliu Hatieganu University of Medicine and Pharmacy, Cluj-Napoca, Romania

2) Morphopathology Department, "Prof. Dr. Ion Chiricuţă" Oncology Institute Cluj-Napoca, Cluj-Napoca, Romania

3) Department of Radiology, "Prof. Dr. Ion Chiricuță" Oncology Institute, Iuliu Hatieganu University of Medicine and Pharmacy, ClujNapoca, Romania
DOI: $10.15386 / \mathrm{mpr}-1604$

Manuscript received: 19.02 .2020

Received in revised form: 26.04 .2020

Accepted: 15.05.2020

Address for correspondence:

r_ciortea@yahoo.com

This work is licensed under a Creative Commons Attribution-NonCommercialNoDerivatives 4.0 International License

\title{
Microscopic distribution of nerve fibers and ganglia within the bladder trigone in women - a cadaveric study
}

\author{
Maria Patricia Rada ${ }^{1}$, Razvan Ciortea $^{1}$, Andrei Mihai Malutan ${ }^{1}$, \\ Bogdan Fetica ${ }^{2}$, Carmen Elena Bucuri ${ }^{1}$, Adelina Clim ${ }^{1}$, Andrei Roman ${ }^{3}$, \\ Dan Mihu ${ }^{1}$
}

\begin{abstract}
Background and aims. The microscopic description of the nerve fibers responsible for micturition is useful when planning minimally invasive interventions for refractory overactive bladder such as intravesical botulinum toxin injections. The purpose of this study was to investigate the density of nerves and ganglia within the bladder trigone, with a focus on identifying areas with a higher density.

Methods. Urinary bladders were harvested from 3 female cadavers. Following tissue processing, a total of 100 slides stained with hematoxylin and eosin (HE) and immunostained for S100 and CD56 were analyzed. The density of nerve fibers (NFD) and ganglia (GD) in 3 different bladder trigone compartments was analyzed.

Results. The NFD in the central compartment (16.2 \pm 3.9$)$ was significantly higher than in both the peripheral $(\mathrm{p}=0.0005)$ and the intermediary $(\mathrm{p}=0.01)$ compartments. The GD was the highest in the peripheral compartment, but it was not significantly different from the other compartments.
\end{abstract}

Conclusions. This microscopic study showed a pattern of distribution with a dominance of nerve fibers in the central compartment and a rather homogenous distribution of the ganglia within the female bladder trigone.

Keywords: nerve endings, ganglia, overactive bladder, botulinum toxin, S100, CD56.

\section{Background and aims}

The nerve fibers emerging from the spinal roots that are responsible for micturition have been described macroscopically. However, their precise microscopic distribution within the bladder wall is not clearly documented, although this would help to better understand the mechanism of micturition.

The innervation of the bladder coordinates a complex mechanism which, in healthy individuals, ensures the balance between two phases of activity: storage and voiding. The muscles involved in micturition receive input from both the autonomic [1] and somatic nervous systems [2]. The motor innervation to the detrusor arises from the parasympathetic ganglion cells in the pelvic plexus $[3,4]$. The preganglionic fibers run in the S2-S4 sacral roots within the pelvic nerves. The sympathetic supply to the bladder arises from the spinal cord segments T12-L2 and runs in the hypogastric plexus. Preganglionic parasympathetic nerves and post-ganglionic sympathetic nerves both synapse close to and within the bladder wall with ganglion cells. The pudendal nerve provides voluntary control over micturition, innervating the external urethral sphincter. In addition to the efferent fibers supplying the bladder, there are sensory fibers that report to the higher centers, running along with 
the motor supply [5]. The proprioceptive afferent fibers enter the sacral segments, similarly to the enteroceptive afferents related to pain and temperature.

The sympathetic and parasympathetic nerve fibers supplying the bladder follow their individual pathways to finally merge in the pelvic plexus [6]. This plexus is located at approximately $1.5 \mathrm{~cm}$ dorso-medially to the ureterovesical junctions, at which point it provides further branches to innervate the vagina and uterus. A group of nerve fibers emerging from the pelvic plexus and terminating directly on the bladder trigone has been previously described [6], but their density in the bladder wall is still to be characterized.

Detailed knowledge of the bladder innervation is important to physicians planning to perform minimally invasive procedures such as intravesical botulinum toxin injections, sacral neuromodulation, percutaneous tibial nerve stimulation in view of treating refractory overactive bladder. It is yet under debate whether the bladder trigone should be spared when performing intravesical botulinum toxin injections, even though recent evidence pleads in favor of the trigone-including technique [7]. We harvested the bladder trigone from female human cadavers with no documented urogenital pathology in order to generate a nerve and ganglia density distribution chart that could be useful when planning the abovementioned procedures.

The purpose of this study was to report nerve and ganglia densities in the female human bladder trigone, with a focus on identifying areas with a higher density.

\section{Methods \\ Ethics considerations}

This study involved the use of human cadaveric tissues. The protocol of the project was approved by our Institutional Ethics Committee (approval number 169/2.4.2018).

\section{Human cadaveric pelvises}

Three fresh, refrigerated human pelvic organ blocks including urinary bladder, distal ureters, uterus and rectum were obtained from our University's Department of Morphologic Sciences, in accordance with the ethical and legal requirements. The samples were preserved at 20 degrees Celsius in $4 \%$ formaldehyde for 3 to 5 days. The causes of death for the females included in the study were intracranial hemorrhage, cardiac arrest and respiratory failure, respectively. All the included subjects were in their 70 's at the time of death and had no medical / surgical history of urogenital pathology, as those were considered exclusion criteria.

\section{Harvesting and processing of human cadaveric urinary bladders}

Storage and further dissection of the specimens were performed at our Institutional Department of Morphologic Sciences.

Preparation of the samples included the dissection of the bladders from the adjacent organs, the opening of the anterior wall of the bladder, harvesting of the trigone, marking of the upper edge of the samples with blue dye, longitudinal sectioning of the trigone into slices at $5 \mathrm{~mm}$ interval and paraffin embedding (Figure $1(\mathrm{~A}-\mathrm{F})$ ).
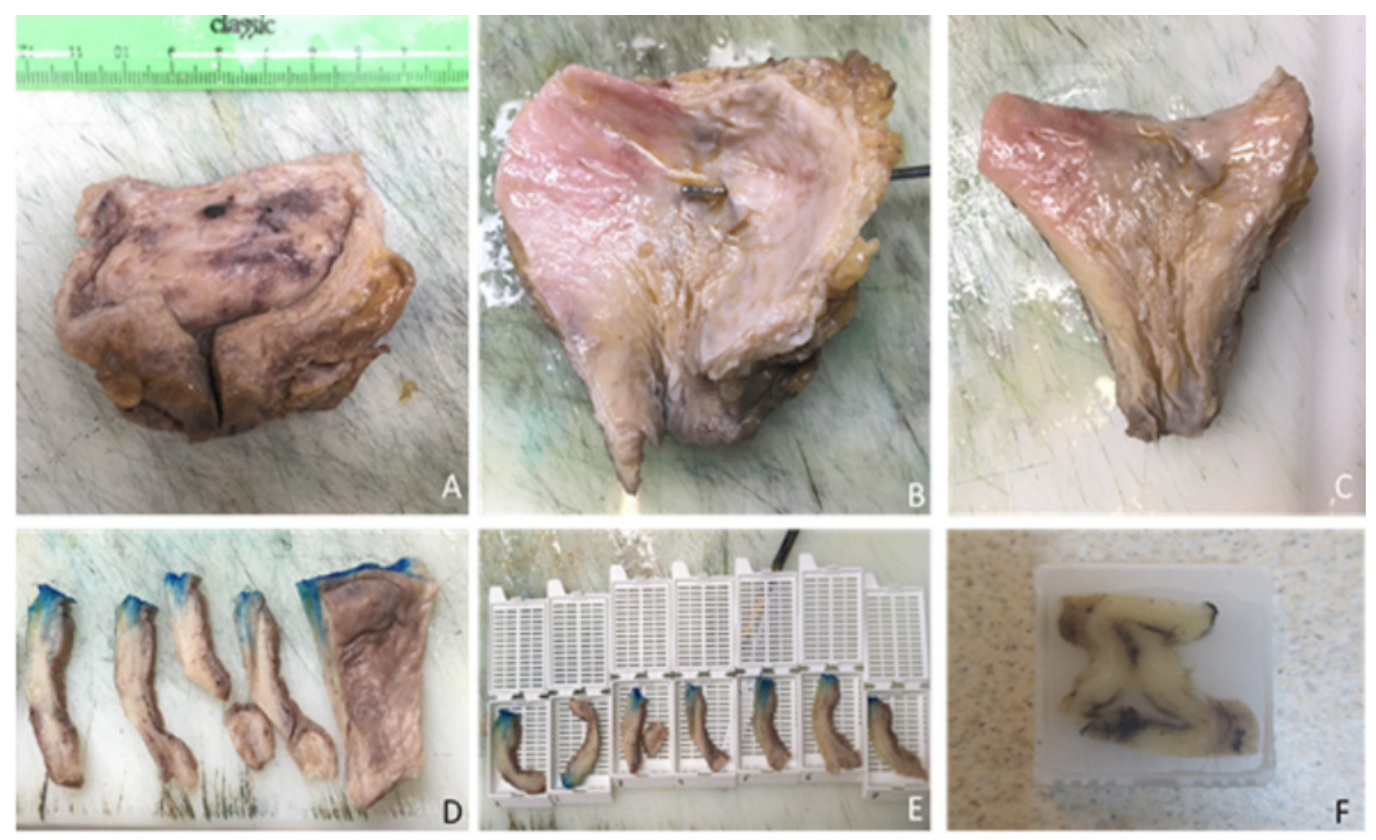

Figure 1. A. Bladder trigone in the process of dissection. B. Bladder trigone with the left ureteric orifice marked. C. Bladder trigone prepared for being sectioned. D. Partially sectioned bladder trigone. E. Preparation of the tissue blocks. F. Paraffin embedded tissues. 
A vertical midline incision in the anterior bladder wall was made in each case and consequently, the internal surface of the bladder was exposed. The ureteric orifices and the interureteric ridges were identified. Dissection of the rectum was then carried out following a plane of dissection along the anterior wall of the rectum. Finally, the bladder trigones were spared respecting the following margins: superiorly, $5 \mathrm{~mm}$ above the interureteric ridge, inferiorly, the boundary between the bladder neck and the urethra and laterally, $5 \mathrm{~mm}$ laterally and parallel to the lines connecting the ureteric orifices and the bladder neck. The superior aspect of the bladder trigone was then marked with blue dye.

The trigone was longitudinally sectioned at $5 \mathrm{~mm}$ and the specimens were subjected to routine procedures for paraffin embedding. Depending on the size of the trigones, a variable number of paraffin blocks were obtained from each case.

\section{Further tissue processing}

In order to obtain the study sections, each paraffin block was serially sectioned at equal intervals and 4 equidistant $2 \mu$ slices were selected for analyses. Half of the sections from each block were stained with hematoxilin and eosin (HE) and the other half was used for immunohistochemistry (IHC), in order to stain the nerve fibers and ganglia.

Two IHC markers (S100 and CD56) were detected using Novocastra Liquid Rabbit Polyclonal Antibody s-100 Protein (product code: NCL-L-S100p) and Novocastra Liquid Mouse Monoclonal Antibody CD56 NCAM (product code: NCL-L-CD56-504), respectively.

\section{Measurements and analysis}

The density of the nerve fibers (NFD) and the ganglia (GD) in different areas of the bladder trigone were the main parameters that have been evaluated. The density was defined as the ratio of the number of nerve fibers or ganglia to the samples' surface expressed in sq.cm.

NFD and GD were assumed to respect midline symmetry. Therefore, the 3 trigones were divided into 6 hemitrigones, which were considered the base unit in our study. In order to analyse the distribution of the nerve fibers and ganglia, each hemitrigone was divided into a peripheral, an intermediary and a central, equidistant, longitudinal compartments (Figure 2).

Depending on the size of the individual trigones, a variable number of paraffin blocks and subsequently a different total number of IHC and HE slices were obtained from each case. A number of 25 slices were immunostained for S100, whereas 50 slices were HE stained. The NFD measurements were compared between the S100 and HE stains. Given that no significant difference in NFD was noted between these groups by using the Mann Whitney test, the subsequent analyses were performed by pooling together the values obtained after analysing both S100 and HE stains.

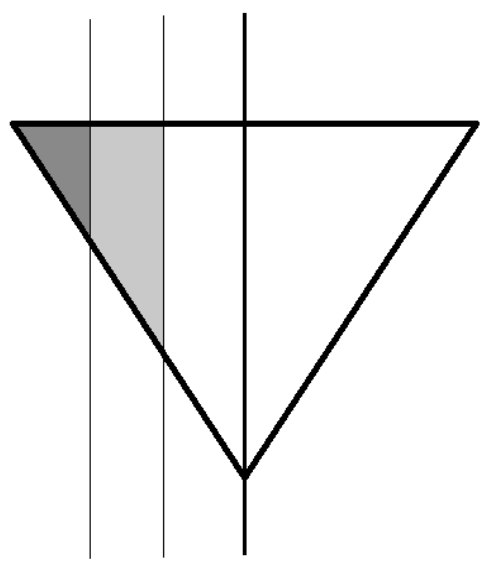

Figure 2. Bladder hemitrigone scheme (dark grey $=$ peripheral compartment, light grey $=$ intermediary compartment, white $=$ central compartment).

Similarly, the ganglia density (GD) was evaluated on 25 slices immunostained for CD56 and on the same HE stained 50 slices. The densities, determined using the two stains were compared and no significant difference was demonstrated. In a similar way as in the case of NFD, CD56 and HE groups were pooled together for further analyses.

The measurements for either NFD or GD resulting from the 6 hemitrigones and the corresponding compartments were pooled together and analyzed using the Kruskal-Wallis test for multiple comparisons.

\section{Results}

Three bladder trigones were processed for the purpose of this study. A total of 100 IHC and HE slices obtained from three different trigone compartments were analyzed.

Figure 3 A-D shows both nerve fibers and ganglia from different trigone compartments, immunostained for S100 and CD 56, respectively.

The NFD measured on HE sections (13.3 \pm 4.2$)$ was not significantly different $(\mathrm{p}=0.1)$ from the NFD measured on sections immunostained for S100 (14 \pm 4.0$)$, allowing both groups to be pooled together for further analysis. The NFD was not significantly different $(p=0.9)$ when the peripheral $(11.8 \pm 3.7)$ and intermediary compartments (13.0 \pm 3.6$)$ were compared. In contrast, the NFD in the central compartment $(16.2 \pm 3.9)$ was significantly higher than in both the peripheral $(p=0.0005)$ and the intermediary ( $\mathrm{p}=0.01$ ) compartment (Figure 4$)$. 

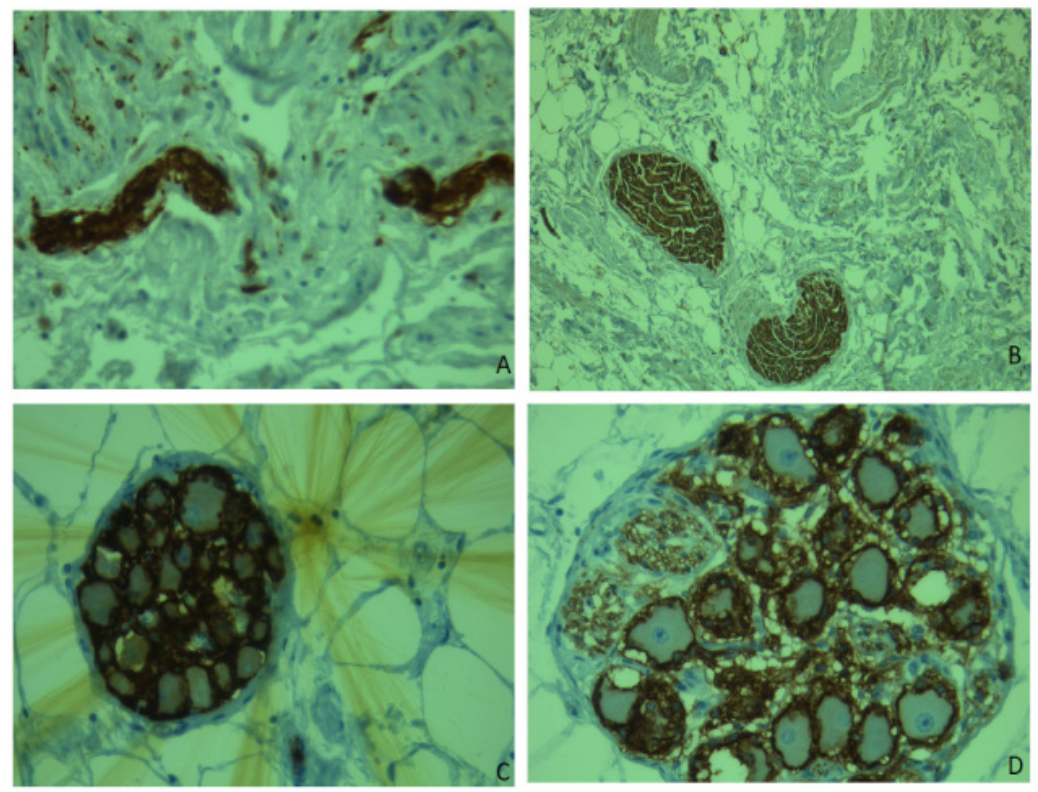

Figure 3. Microscopic images of the specimens. A. nerve fiber immunostained for S100, longitudinal section. B. nerve fiber immunostained for S100, transverse section. C, D. ganglia, immunostained for CD56.

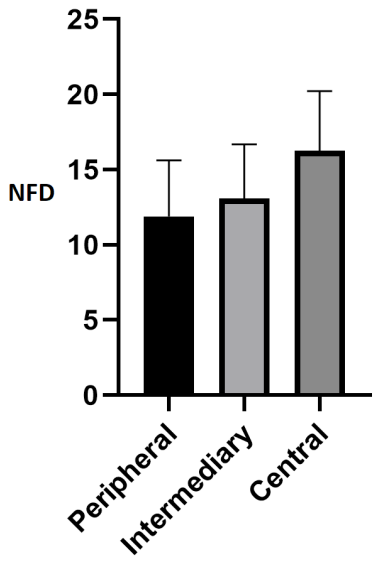

Figure 4. NFD in the peripheral, intermediary, and central bladder trigone compartments.

The GD was lower when measured on HE sections $(0.4 \pm 0.4)$ than when measured on IHC marked sections $(0.6 \pm 0.5)$, but the difference was not statistically significant $(p=0.1)$, therefore the two groups were analyzed together. The GD was highest in the peripheral compartment $(0.6 \pm 0.4)$, followed by the central $(0.5 \pm 0.4)$ and intermediary $(0.4 \pm 0.5)$ compartment, respectively. However, there was no significant difference at the multiple comparisons of the following compartments: peripheral vs. intermediary $(\mathrm{p}=0.1)$; peripheral vs. central $(\mathrm{p}=0.9)$; intermediary vs. central $(\mathrm{p}=0.9)$ (Figure 5).

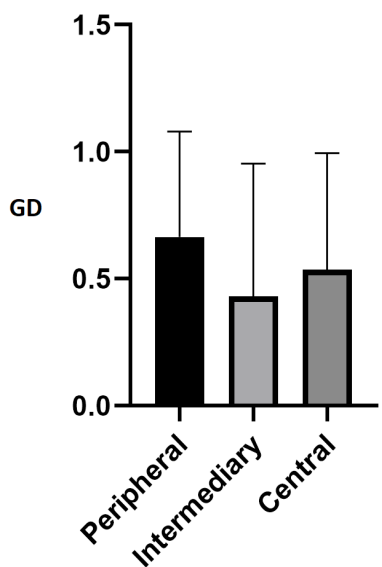

Figure 5. GD in the peripheral, intermediary and central bladder trigone compartments.

\section{Discussion}

The NFD distribution chart generated based on the findings of this study have confirmed that the human female trigone is most densely innervated in the central compartment. Some reports previously highlighted neuroanatomical relationships aspects specific to bladder neck area [8]. More precisely, it has been shown that the highest density of autonomic nerve fibers was at the level of the posterior aspect of the bladder neck, at 4 and 8 o'clock positions, in specimens harvested from women $[8,9]$. These areas fall within the region defined as the 
central compartment in this study. The focus of previous research on the area of the bladder neck was most likely triggered by surgical concerns due to the risk of denervation and devascularization of this structure during surgical dissection, when performing procedures for stress urinary incontinence or pelvic organ prolapse.

Due to the complexity and significant number of nerve fibers innervating the bladder, it is challenging to track the precise course of individual nerves. Alternatively, investigating their density may be beneficial when considering treatment methods for overactive bladder such as intradetrusor botulinum toxin injections. Optimal sites of injection are still to be determined. Currently, most physicians distribute the injections into the bladder wall, sparing the trigone $[10,11]$, even though more recent research studies have shown that the trigone-including technique does not entail greater complication rates and has a good efficacy and safety profile [12]. The findings of our study may be helpfulwhen planning the injection sites pattern, given that we demonstrated that there are compartments with higher NFD.

While it has been reported that, in other species, autonomic ganglia concentrate around the ureterovesical junctions [13-16], which correspond to the peripheral compartment in our study, in humans there are doubts concerning their distribution in the same areas, as well as in other regions of the human bladder wall such as the bladder trigone. Some authors have reported a lack of ganglia in the human bladder dome and lateral walls $[17,18]$. Further evidence demonstrated the presence of clusters of ganglion cells within the bladder wall [13], which were widely spread. However, the trigone itself was rarely investigated and this site has drawn attention more recently, once the question whether the trigone should be spared or not when injecting intravesical botulinum toxin for refractory overactive bladder arose. The main strength of our study is that the focus has been particularly on the trigone, rather than on the entire bladder wall. We demonstrated that the ganglia density was the highest in the peripheral compartment of the trigone, but not significantly higher than in the other compartments. The practical importance of the intramural ganglia resides on the fact that their presence will maintain some autonomous nervous function despite sectioning of the pelvic nerves that occurs during surgery [19].

Besides intravesical botulinum toxin injections, other minimally invasive treatments for refractory overactive bladder such as sacral neuromodulation and percutaneous tibial nerve stimulation have successfully improved lower urinary tract symptoms in cases where the conservative measures have failed [20,21]. Complications associated with these procedures include but are not limited to pain, infection, bleeding or neuropathy [21]. Mapping of the targeted nerves may refine and improve the treatment strategies, assisting at the development of novel approaches.
Most studies reporting on bladder wall innervation used tissues from subjects with urinary tract pathology $[22,23]$, which may lead to results that are not reflecting the normal anatomy. Moreover, histological observations were mostly based on biopsy samples [24], obtained by cystoscopy, rather than on the bladder as a whole or just a specific region of the bladder. Our study investigated the human trigone, harvested from female cadavers with no documented urogenital pathology. However, any generalization of the study results should be cautious, considering the limited number of specimens and sections that have been examined.

In addition, we have only studied the NFD and GD in trigones harvested from postmenopausal cadaveric women and it is known that overactive bladder has an increased prevalence in this population [25]. It is therefore possible that differences in the NFD and GD of women of reproductive age, pre- or perimenopausal exist. Future research that may explore this fact, aiming to compare tissue samples harvested from women in different biological life stages, with and without uro-gynecological pathology could help clarify this issue.

Another limitation is related to the slightly lower detection rate of the nerve fibers and ganglia on HE stains. However, given that we demonstrated that the difference was not statistically significant, we assumed that the impact on the results was minimal. Another aspect that should be considered is that $\mathrm{S} 100$ protein is only present in the myelinated fibers, and therefore, smaller, unmyelinated fibers such as C-fibers, which carry sensory information have not been detected in our study. Finally, the actual nerve topography of the bladder may be slightly different in vivo conditions, as tissue harvesting and preservation may carry the inherent risk of alteration of samples.

\section{Conclusions}

This microscopic study on samples harvested from female human cadavers showed a pattern of nerves and ganglia distribution with a dominance of nerve fibers in the central compartment and a rather homogenous distribution of the ganglia within the bladder trigone.

\section{Acknowledgements}

This study was funded by Iuliu Hațieganu University of Medicine and Pharmacy Cluj-Napoca, grant number 1680/74. The authors would like to thank Dr Anca Rasca, Dr Larisa Pop, laboratory assistants Ioana Cilean and Rafila Bara for helping with tissue sampling and preparation of the slices.

\section{References}

1. Hadley G. Essential clinical anatomy. J Anat. 2007;211:413.

2. Chancellor MB, Yoshimura N. Neurophysiology of stress 
urinary incontinence. Rev Urol. 2004;6:S19-S28.

3. Baggish MS, Karram MM. Atlas of pelvic anatomy and gynecologic surgery: Elsevier Health Sciences; 2015.

4. Fowler CJ, Griffiths D, de Groat WC. The neural control of micturition. Nat Rev Neurosci. 2008;9:453-66.

5. Wyndaele J, De Wachter S. The basics behind bladder pain: a review of data on lower urinary tract sensations. Int J Urol. 2003;10:S49-S55.

6. Purves JT, Spruill L, Rovner E, Borisko E, McCants A, Mugo E, et al. A three dimensional nerve map of human bladder trigone. Neurourol Urodyn. 2017;36:1015-9.

7. Manecksha RP, Cullen IM, Ahmad S, McNeill G, Flynn R, McDermott TE, et al. Prospective randomised controlled trial comparing trigone-sparing versus trigone-including intradetrusor injection of abobotulinumtoxinA for refractory idiopathic detrusor overactivity. Eur Urol. 2012;61:928-935.

8. Spradling K, Khoyilar C, Abedi G, Okhunov Z, Wikenheiser $\mathrm{J}$, Yoon R, et al. Redefining the Autonomic Nerve Distribution of the Bladder Using 3-Dimensional Image Reconstruction. J Urol. 2015;194:1661-1667.

9. Ball TP Jr, Teichman JM, Sharkey FE, Rogenes VJ, Adrian EK Jr. Terminal nerve distribution to the urethra and bladder neck: considerations in the management of stress urinary incontinence. J Urol. 1997;158:827-829.

10. Apostolidis A, Rahnama'i MS, Fry C, Dmochowski R, Sahai A. Do we understand how botulinum toxin works and have we optimized the way it is administered to the bladder? ICIRS 2014. Neurourol Urodyn. 2016;35:293-298.

11. Jiang YH, Kuo HC. Reduction of urgency severity is the most important factor in the subjective therapeutic outcome of intravesical onabotulinumtoxinA injection for overactive bladder. Neurourol Urodyn. 2017;36:338-343.

12. Jo JK, Kim KN, Kim DW, Kim YT, Kim JY, Kim JY. The effect of onabotulinumtoxinA according to site of injection in patients with overactive bladder: a systematic review and meta-analysis. World J Urol. 2018;36:305-317.

13. Dixon JS, Gilpin SA, Gllpln CJ, Gosling JA. Intramural ganglia of the human urinary bladder. Br J Urol. 1983;55:195198.

14. Schulman CC, Duarte-Escalante O, Boyarsky S, Gregoir W. New concepts of ureterovesical innervation. J Urol. 1973; 109:381-384.
15. Zhao Z, Xu Q, Lu L, Luo X, Fu X. Intramuscular nerve distribution in bladder and the relationship between intramuscular ganglia and bladder function in man and dog. Int J Clin Exp Med. 2014; 7:5083-5089.

16. Montalbetti N, Rued AC, Taiclet SN, Birder LA, Kullmann FA, Carattino MD. Urothelial Tight Junction Barrier Dysfunction Sensitizes Bladder Afferents. eNeuro. 2017; 24;4(3):ENEURO.0381-16.2017.

17. Ek A, Alm P, Andersson KE, Persson CG. Adrenergic and Cholinergic Nerves of the Human Urethra and Urinary Bladder. A histochemical study. Acta Physiol Scand. 1977;99:345-352.

18. Sundin T, Dahlström A, Norlén L, Svedmyr N. The sympathetic innervation and adrenoreceptor function of the human lower urinary tract in the normal state and after parasympathetic denervation. Invest Urol. 1977;14:322-328.

19. Fugett J 2nd, Phillips L, Tobin E, Whitbrook E, Bennett H, Shrout J, et al. Selective bladder denervation for overactive bladder (OAB) syndrome: From concept to healing outcomes using the ovine model. Neurourol Urodyn. 2018;37:20972105 .

20. McCrery R, Lane F, Benson K, Taylor C, Padron O, Blok $\mathrm{B}$, et al. Treatment of Urinary Urgency Incontinence Using a Rechargeable SNM System: 6-Month Results of the ARTISAN-SNM Study. J Urol. 2020;203(1):185-192.

21. Ammirati E, Giammò A, Manassero A, Carone R. Neuromodulation in urology, state of the art. Urologia. 2019;86:177-182.

22. Traini C, Del Popolo G, Faussone-Pellegrini MS, Guasti D, Catarinicchia S, Vannucchi MG. Nerve sprouting and neurogenic inflammation characterize the neurogenic detrusor overactive bladder of patients no longer responsive to drug therapies. J Cell Mol Med. 2019;23:4076-4087.

23. Compérat E, Reitz A, Delcourt A, Capron F, Denys P, Chartier-Kastler E. Histologic features in the urinary bladder wall affected from neurogenic overactivity--a comparison of inflammation, oedema and fibrosis with and without injection of botulinum toxin type A. Eur Urol. 2006;50:1058-1064.

24. Kuijpers KA, Heesakkers JP, Schalken JA. Alterations of the myovesical plexus of the human overactive detrusor. Biomed Res Int. 2014;2014:754596.

25. Tomaszewski J. Postmenopausal overactive bladder. Prz Menopauzalny. 2014;13:313-329. 